\title{
Laboratory Changes in SARS-CoV-2 Infection: A Review
}

\author{
Devesh Sharma ${ }^{1}$, Mohit Mehndiratta ${ }^{2}$, Dinesh Puri ${ }^{3}$
}

\begin{abstract}
Aim: To review the updated literature to find out the biochemical and hematological changes that occur in the SARS CoV-2 (COVID-19) infection that is causing the ongoing global pandemic.

Background: The outbreak of COVID-19 infection began in December 2019 in Wuhan, Hubei province, and within a few months, by March 2019 , it had taken the shape of a pandemic. Six months down the road, knowledge about the pathogenesis of this infection has grown rapidly. Although it is predominantly a respiratory infection, COVID-19 is now known to affect almost every organ of the body. The unpredictable clinical course has been attributed to multiple biochemical and hematological changes that are caused by the COVID-19 virus.

Review results: An in-depth analysis of recently published data has highlighted that various biochemical and hematological alterations can be attributed to COVID-19 infection. These include hypoalbuminemia, reduced number of lymphocytes, elevated levels of aspartate transaminase (AST), homocysteine, and biomarkers such as ferritin. Other parameters such as lactate dehydrogenase (LDH), C-reactive protein (CRP), IL-6, Procalcitonin (PCT), D-Dimer, neutrophil-lymphocyte ratio (NLR), and angiotensin II correlate with the extent of viral load and predicted the course of the disease.

Conclusion: Knowledge about the alteration biochemical and hematological markers in the COVID-19 infection is helpful in the estimation of the extent of viral load and in the prediction of the clinical course of the infection.

Clinical significance: Monitoring the biochemical and hematological biomarkers, especially, CRP, ferritin, LDH, D-Dimer, and NLR, is useful for triaging patients infected with COVID-19 early in the course of disease and thus may prevent disease progression by the institution of early interventions.

Keywords: Acute respiratory distress syndrome, Biochemical and hematological changes, COVID-19, Pandemic, SARS-CoV-2.

Indian Journal of Medical Biochemistry (2020): 10.5005/jp-journals-10054-0142
\end{abstract}

\section{BACKGROUND}

The novel, severe acute respiratory syndrome coronavirus 2 (SARS-CoV-2) virus outbreak was first identified in Wuhan, China, in December 2019. Within 6 months, the outbreak took on the shape of a pandemic which has now affected more than 64 million people across the globe and caused nearly 1.5 million deaths. ${ }^{1}$ The main mechanism for infection with SARS-CoV-2 virus is the binding of the spike protein of the virus to the membrane-bound form of angiotensin-converting enzyme 2 (ACE2) and the internalization of complex by the host cell. ACE2 receptors are not only expressed in the lung but also in the small intestines, colon, esophagus, heart, kidney, ovaries, and testis. The widespread distribution of receptors of SARS-CoV-2 virus is the reason behind the multiple clinical manifestations of infection as well as multiorgan damage and systemic failure in advanced stages of infection.

The clinical course of SARS-CoV-2 is mild in most cases $(80 \%)$, whereas in the remaining $(20 \%)$, the disease can vary from moderate (15\%) to severe $(5 \%)$ ends of the spectrum. ${ }^{2}$ Progression of infection in the spectrum of moderate-to-severe disease was very unpredictable at the onset of the pandemic. However, as the volume of scientific data available has increased over the period of 6 months down the pandemic, the role of biomarkers in the timely triage of patients has gained importance. In categorization of patients based on the extent of disease severity, biomarkers are proving to play an important role in optimum utilization of the resources during the pandemic. ${ }^{2}$ All studies till now have persistently described that, in severe infection by SARS-CoV-2 virus, there is underlying damage to organs ensued by the activation of the complement system, inflammatory cascades, and proinflammatory cytokines (IL-6). Biomarkers, therefore, help in the evaluation of cellular and organ
${ }^{1-3}$ Department of Biochemistry, University College of Medical Sciences and GTB Hospital, New Delhi, India

Corresponding Author: Dinesh Puri, Department of Biochemistry, University College of Medical Sciences and GTB Hospital, New Delhi, India, Phone: +919868838960, e-mail: dineshpuri21@yahoo.co.in

How to cite this article: Sharma D, Mehndiratta M, Puri D. Laboratory Changes in SARS-CoV-2 Infection: A Review. Indian J Med Biochem 2020;24(2):62-65.

Source of support: Nil

Conflict of interest: None

damage caused by SARS-CoV-2 virus. Therefore, these biomarkers play an indispensable role in not just clinical management but also in the timely screening of those patients who are on the trajectory to develop severe disease. ${ }^{3}$ The process of vasculitis results in thromboembolism, which in turn leads to both lung damage [acute respiratory distress syndrome (ARDS)] and cardiovascular (ischemia and pulmonary thromboembolism) and cerebrovascular damage. Biomarkers such as D-Dimer help in the prediction of these vascular complications, but they have their own limitations. ${ }^{4}$

Apart from routine investigations such as AST, markers of organ damage such as LDH and inflammatory mediators like IL- 6 help in the identification of those patients who are at risk of development of fatal complications, especially due to cytokine storm, where anti-IL- 6 therapy like tocilizumab are showing some benefit in reducing mortality. ${ }^{5}$ Markers of systemic inflammation such as ferritin and CRP have an important role to play in a country like India, where IL- 6 cannot be so widely estimated due to the high cost of the test. ${ }^{2}$ 
The biomarkers such as D-dimer, prothrombin time (PT), and fibrin degradation products (FDP) also play an important role in the prevention of complications due to the thrombotic state induced by SARS-CoV-2 infection. They help in the process of decision-making on whether anticoagulant therapy should be started early in the course of the disease. The biomarkers such as CRP, LDH, creatine kinase (CK), ferritin, and IL-6 along with hematological parameters, such as white blood cell count (WBC), neutrophil-lymphocyte ratio (NLR), and monocyte-lymphocyte ratio (MLR), play an important role as criteria in the classification of the disease process as mild, moderate, or severe. ${ }^{6}$ Recently, another biomarker, homocysteine, has also been reported as a predictor of the increased risk of cardiovascular complications in those patients who present with severe pneumonia on computed tomography scan during the first week of COVID infection. ${ }^{7}$ This review is directed at the current state of knowledge regarding the known as well as repurposed biomarkers in the assessment of the severity of SARS-CoV-2 infection. These have proven to play a pivotal role in the prediction of organ damage and thus in the prevention of severe complications and death. ${ }^{8}$

\section{Review Results}

Laboratory parameters that are altered in COVID-19 infection include biochemical and hematological parameters, along with immunological and inflammatory markers. These values are significantly different in patients with mild disease compared to those with severe disease. These markers help in risk assessment and in the categorization of patients for timely clinical interventions.

\section{Biochemical Markers}

Yang et al. compared the biochemical changes in survivors and nonsurvivors in COVID-19 infection. They found a significant increase in serum levels of ferritin, total bilirubin, CK, and IL-6 in the latter group. ${ }^{9}$

The markers of cardiac injury (cardiac troponins) were found to be significantly elevated at the time of presentation in nonsurvivors when compared to survivors of COVID-19 infection. This was also true for markers of muscular and tissue injury, such as LDH. In cases with multiple organ failure, there was a significant derangement in liver function tests (LFT) and especially in the elevation in AST and ALT and renal function tests (RFT). The RFT also showed critical elevations in blood urea nitrogen and creatinine levels. ${ }^{6}$ In one of the studies, it was reported that there were significantly higher levels of $\mathrm{LDH}, \mathrm{CK}, \mathrm{N}$-terminal pro Brain Natriuretic peptide (NT-ProBNP), ALT, AST, and creatinine in the non-survivor group. It was also found that when compared to other cases of viral hepatitis where ALT levels are a marker of liver dysfunction, elevation in AST was a more specific maker of SARS-CoV-2-induced liver injury and correlated with the mortality risk. ${ }^{10}$ For patients with severe COVID-19 infection, levels of CRP (an inflammatory marker) were found to be increased. These levels were significantly elevated in the initial phases of infection, even before the appearance of critical findings in the CT. Apart from CRP, the severity of CT scores also correlated positively with erythrocyte sedimentation rate (ESR), NLR. ${ }^{11}$ Inflammatory markers such as ferritin were found to be significantly increased in nonsurvivors when compared to survivors (mean: $4600 \mathrm{ng} / \mathrm{mL}$ and 760 $\mathrm{ng} / \mathrm{mL}$ respectively). In advanced stages of COVID-19 infection, there is a cytokine storm, and it is accompanied by the elevation in other inflammatory mediators, such as IL-2, IL-7, tumor necrosis factor (TNF-alfa), monocyte chemoattractant protein (MCP)-1, CRP, procalcitonin $(\mathrm{PCT})$, and granulocyte colony-stimulating factor (G-CSF) along with IL-6 and ferritin. ${ }^{12,13}$ PCT was markedly raised in patients with severe disease. Increased PCT levels also correlated with a higher risk for severe SARS-CoV-2 infection (OR: 4.76). ${ }^{14}$

The hematological biomarkers that are used to categorize patients and in the evaluation of the risk for critical COVID infection include, total leukocyte count (TLC), meutrophil count, lymphocyte count, NLR, and platelet count. Lymphopenia was found to be present in $80 \%$ of patients with severe disease. ${ }^{9}$ Apart from lymphopenia, severe cases of COVID-19 infection tended to have high levels of NLR, thrombocytopenia, monocytopenia, and eosinopenia. ${ }^{6}$ Very low eosinophil counts at the time of admission were found to indicate a poor prognosis. ${ }^{15}$ The changes in lymphocyte population in severe COVID-19 infection included an increase in the number of naive T-helper cells and a decrease in the number of memory T-cells, along with total NK cells, T cells, and $B$ cells. ${ }^{16}$ NLR has been identified as an independent risk factor for severe disease. NLR of 3 or more than 3 , at the time of hospitalization, predicted a greater chance of progression to severe disease. ${ }^{17}$ Platelet counts were found to be significantly reduced in the COVID-19 patients who did not survive and was also found to be indicator of worsening during hospitalization. It was also observed that patients with a higher thrombocytes to lymphocytes ratio had a longer duration of hospitalization. ${ }^{18}$

\section{Coagulation Parameters}

Derangement of coagulation parameters correlated with a poorer prognosis. The level of D-dimer was found to be frequently elevated in patients with SARS-CoV-2 infection and to some extent predicted the progression to severe disease. ${ }^{19}$ Wang et al. had reported that levels of D-dimer and thrombin time were elevated in the nonsurvivor group, whereas aPTT was found to be lower in this group when compared to the survivor group. They also reported that more than half of the patients in their study had a prolonged prothrombin time (PT). ${ }^{20}$ These findings were corroborated by another study in which, along high D-dimer and prolonged PT, fibrin degradation products (FDP) were also found to be elevated in the non-survivor group. ${ }^{21}$

\section{Discussion}

The understanding of pathophysiological basis of alteration of various biomarkers has increased over time during the ongoing pandemic. The main pathophysiological mechanism for infection with SARS-CoV-2 is the binding of the virus to the membranebound form of angiotensin-converting enzyme 2 (ACE2) and the subsequent internalization of the complex. ${ }^{22}$

The derangement of liver function in COVID-19 has been mainly attributed to the binding of SARS-CoV-2 to ACE-2-positive cholangiocytes, causing functional abnormalities in cholangiocyte function. In advanced stages of infection, the liver injury induced by the systemic inflammatory response leads to the elevation of liver enzymes.

The increase in inflammatory markers, such as CRP and Ferritin, mark the onset as well as progression of the systemic vasculitis and coagulation abnormalities, which, in turn, lead to damage to the vital organs. The increase in inflammatory markers such as IL-6 mark the onset of the cytokine-storm and subsequent acute lung injury, leading to ARDS and eventually to multiple organ failure and death. ${ }^{13}$ 
Procalcitonin (PCT) level is another inflammatory mediator, which is increased in severe infections caused by bacteria, parasites, and fungi that cause systemic disease leading to the production of procalcitonin from extra thyroid tissue. ${ }^{23}$

In contrast, interferon synthesized in response to viral infection suppresses the synthesis of procalcitonin by downregulation. This offers the advantage of procalcitonin assay to distinguish between bacterial infections and the systemic inflammatory response due to viral infections such as COVID-19. ${ }^{24}$ Therefore, the initial value of PCT could help in the diagnosis of severe illness.

Among hematological changes, lymphopenia correlated strongly with the severity of illness, especially the number of regulatory T-lymphocytes, which were low in such cases. ${ }^{25}$ Cytotoxic lymphocytes and natural killer cells play a crucial role in the control of the spread of viral infection. Therefore, in severe disease, there is exhaustion of cytotoxic T-lymphocytes which correlate with disease progression. ${ }^{26}$

Another hematological marker of disease severity was NLR. It is also an inflammatory marker and has been shown to predict the probability of death in various cardiovascular diseases. ${ }^{27}$ Elevation in NLR is due to the dysregulated expression of inflammatory cytokines caused by SARS-CoV-2 infection, which leads to an increase in neutrophils and an enhanced expression of the genes involved in the lymphocytic apoptotic pathway. ${ }^{28}$

It has also been postulated that COVID-19 infection can directly hamper normal hematopoiesis by affecting the precursors of various cells in the bone marrow. ${ }^{29}$ Likewise, the platelet count has also been found to serve as an easily available biomarker of the risk of mortality in COVID-19 patients in intensive care units. Platelet count is reduced in COVID-19 infection due to the increased consumption of platelets as a result of platelet aggregation and the formation of microthrombi due to pulmonary endothelial damage. ${ }^{30}$

In severe cases of COVID-19 infection, disseminated intravascular coagulation (DIC) occurs due to the release of cytokines from monocytes and endothelial cells following cell injury.

Circulating levels of free thrombin activate platelets and causes fibrinolysis, leading to an increase in the level of $\mathrm{D}$-dimer and fibrin degradation products. These were found to be markedly elevated in all cases of death due to COVID-19. This suggests that it is a disease in which there is activation of coagulation, causing a rapid progression of the disease, which is nonresponsive to mechanical ventilation, and there is rapid and positive response in some critically ill patients given anticoagulation therapy. ${ }^{31}$

\section{Conclusion}

Since the beginning of the pandemic, there has been a significant improvement in the management of patients affected by COVID19 due to an improved understanding of the pathophysiology and the biomarkers reflecting it. From the published studies, it can be concluded that severe SARS-CoV-2 infection can be predicted by alterations in hematological biomarkers, such as lymphopenia, neutrophilia, and an increase in NLR; biochemical markers such as $\mathrm{LDH}$, Ferritin, CRP, and AST; and coagulation parameters such as $\mathrm{D}$-Dimer and FDP. This is now leading to a shift in the management of patients based on not just clinical symptoms but also the profiles of these biomarkers. This helps in the early stratification of the risk to the patient and the optimal allocation of limited resources of health care, which are strained due to the large number of cases of SARS-CoV2.
As per changes in the levels of these biomarkers, protocols have now improved over the period of nearly 1 year for a more specific, supportive management of the patient. These measures include timely institution of anticoagulation therapy with low-molecularweight heparin and anti-inflammatory medicines such as NSAIDs and dexamethasone. In severe cases, IL-6 receptor blockers like Toclizumab have proved to be lifesaving.

For better clinical management of the patient, clinical scores can be developed using biomarkers and clinical parameters, which can help in the individualization of treatment to the patients based on the severity of their disease. There is a huge scope to explore other biomarkers that can also help in the better understanding of pathophysiology and management of the new corona virus that has affected the whole world in a very short period of time.

\section{Acknowledgments}

The authors would like to thank all those patients who could survive and also who could not survive the onslaught of COVID-19 pandemic and the investigators who have contributed the data for this review. We are also thankful to all the healthcare workers including the scientists who have mitigated the severity of this pandemic by the development and improvement of the diagnostic modalities for COVID-19.

\section{References}

1. @OurWorldInData Total confirmed COVID-19 deaths: how rapidly are they increasing?: @OurWorldInData, 2020. Available: https:// ourworldindata.org/grapher/covid-confirmed-deaths-since-5thdeath.

2. Esakandari H, Nabi-Afjadi M, Fakkari-Afjadi J, et al., A comprehensive review of COVID-19 characteristics. Biol Proced Online [Internet]. 2020 Aug 4 [cited 2020 Sep 24];22. Available from: https://www.ncbi.nlm. nih.gov/pmc/articles/PMC7402395/.

3. Lin L, Lu L, Cao W, et al. Hypothesis for potential pathogenesis of SARS-CoV-2 infection-a review of immune changes in patients with viral pneumonia. Emerg Microbes Infect 2020;9(1):727-732. DOI: 10.1080/22221751.2020.1746199.

4. Clinical features of patients infected with 2019 novel coronavirus in Wuhan, China - PubMed [Internet]. [cited 2020 Sep 25]. Available from: https://pubmed.ncbi.nlm.nih.gov/31986264/.

5. Cytokine release syndrome in severe COVID-19: interleukin-6 receptor antagonist tocilizumab may be the key to reduce mortality- PubMed [Internet]. [cited 2020 Sep 25]. Available from: https://pubmed.ncbi. nlm.nih.gov/32234467/.

6. Henry BM, de Oliveira MHS, Benoit S, et al. Hematologic, biochemical and immune biomarker abnormalities associated with severe illness and mortality in coronavirus disease 2019 (COVID-19): a meta-analysis. Clin Chem Lab Med CCLM 2020;58(7):1021-1028. DOI: 10.1515/cclm2020-0369.

7. Yang Z, Shi J, He Z, et al. Predictors for imaging progression on chest CT from coronavirus disease 2019 (COVID-19) patients. Aging 2020;12(7):6037-6048. DOI: 10.18632/aging.102999Google Search [Internet]. [cited 2020 Sep 25]. Available from: https://www.google. $\mathrm{com} /$ search?q=Yang $+\mathrm{Z} \% 2 \mathrm{C}+\mathrm{Shi}+\mathrm{J} \% 2 \mathrm{C}+\mathrm{He}+\mathrm{Z} \% 2 \mathrm{C}+\mathrm{et}+\mathrm{al} .+$ Predicto $\mathrm{rs}+$ for+imaging+progression+on+chest+CT+from+coronavirus+d isease+2019+(COVID-19)+patients.+Aging.+2020\%3B12\%3A6037\% E2\%80\%936048.\&oq=Yang $+Z \% 2 \mathrm{C}+\mathrm{Shi}+\mathrm{J} \% 2 \mathrm{C}+\mathrm{He}+\mathrm{Z} \% 2 \mathrm{C}+\mathrm{et}+\mathrm{al} .+\mathrm{P}$ redictors+for+imaging+progression+on+chest+CT+from+corona virus+disease+2019+(COVID-19)+patients.+Aging.+2020\%3B12\%3 A6037\%E2\%80\%936048.\&aqs=chrome.69i57.24083j0j4\&sourceid $=$ chrome\&ie=UTF-8.

8. Characterization of the receptor-binding domain (RBD) of 2019 novel coronavirus: implication for development of RBD protein as a viral attachment inhibitor and vaccine | Cellular \& Molecular Immunology 
[Internet]. [cited 2020 Sep 25]. Available from: https://www.nature. com/articles/s41423-020-0400-4.

9. Yang $X, Y u Y, X u$ J, et al. Clinical course and outcomes of critically ill patients with SARS-CoV-2 pneumonia in Wuhan, China: a singlecentered, retrospective, observational study. Lancet Respir Med 2020;8(5):475-481. DOI: 10.1016/S2213-2600(20)30079-5.

10. Lei F, Liu Y-M, Zhou F, et al. Longitudinal association between markers of liver injury and mortality in COVID-19 in China. Hepatology 2020;72(2):389-398. DOI: 10.1002/hep.31301.

11. Tan C, Huang $Y$, Shi F, et al. C-reactive protein correlates with CT findings and predicts severe COVID-19 early. J Med Virol 2020;92(7):856-862. DOI: 10.1002/jmv.25871.

12. Wang D, Hu B, Hu C, et al. Clinical characteristics of 138 hospitalized patients with 2019 novel coronavirus- infected pneumonia in Wuhan, China. JAMA 2020;323(11):1061-1069. DOI: 10.1001/jama.2020. 1585.

13. Mehta P, McAuley DF, Brown M, et al. COVID-19: consider cytokine storm syndromes and immunosuppression. Lancet 2020;395(10229):1033-1034. DOI: 10.1016/S0140-6736(20)30628-0.

14. Lippi G, Plebani M. Procalcitonin in patients with severe coronavirus disease 2019 (COVID-19): a metaanalysis. Clin Chim Acta 2020;505:190191. DOI: 10.1016/j.cca.2020.03.004.

15. Du R-H, Liang L-R, Yang C-Q, et al. Predictors of mortality for patients with COVID-19 pneumonia caused by SARS-CoV-2: a prospective cohort study. Eur Respir J 2020;55(5):2000524. DOI: 10.1183/13993003.00524-2020.

16. Cossarizza A, De Biasi S, Guaraldi G, et al. SARS-CoV-2, the virus that causes COVID-19. Cytometry 2020;97(4):340-343. DOI: 10.1002/ cyto.a.24002.

17. Long L, Zeng X, Zhang X, et al. Short-term outcomes of coronavirus disease 2019 and risk factors for progression. Eur Respir J 2020;55(5):2000990. DOI: 10.1183/13993003.00990-2020.

18. Lippi G, Plebani M, Henry BM. Thrombocytopenia is associated with severe coronavirus disease 2019 (COVID-19) infections: a meta-analysis. Clin Chim Acta 2020;506:145-148. DOI: 10.1016/ j.cca.2020.03.022.
19. Lippi G, Favaloro EJ. D-dimer is associated with severity of coronavirus disease 2019: a pooled analysis. Thromb Haemost 2020;120(5):876878. DOI: 10.1055/s-0040-1709650.

20. Wang $\mathrm{K}$, Zuo $\mathrm{P}$, Liu $\mathrm{Y}$, et al. Clinical and laboratory predictors of in-hospital mortality in patients with COVID-19: a cohort study in Wuhan, China. Clin Infect Dis 2020. ciaa538. DOI: 10.1093/cid/ciaa538.

21. Tang N, Li D, Wang X, et al. Abnormal coagulation parameters are associated with poor prognosis in patients with novel coronavirus pneumonia. J Thromb Haemost 2020;18(4):844-847. DOI: 10.1111/ jth.14768.

22. Donoghue $\mathrm{M}, \mathrm{Hsieh} F$, Baronas $\mathrm{E}$, et al. A novel angiotensin-converting enzyme-related carboxypeptidase (ACE2) converts angiotensin I to angiotensin 1-9. Circ Res 2000;87(5):E1-E9. DOI: 10.1161/01.RES.87.5.e1.

23. Russwurm S, Wiederhold M, Oberhoffer M, et al. Molecular aspects and natural source of procalcitonin. Clin Chem Lab Med 1999;37(8):789-797. DOI: 10.1515/CCLM.1999.119.

24. Cleland DA, Eranki AP. Procalcitonin. StatPearls; [Internet]. Treasure Island (FL): StatPearls Publishing; 2020.

25. Qin $\mathrm{C}$, Zhou L, Hu Z, et al. Dysregulation of immune response in patients with COVID-19 in Wuhan, China. Clin Infect Dis 2020(15). DOI: $10.1093 /$ cid/ciaa248.

26. Lippi G, Henry BM. Eosinophil count in severe coronavirus disease 2019 (COVID-19). QJM Mon J Assoc Phys 2020;113(7):511-512.

27. Bhat T, Teli S, Rijal J, et al. Neutrophil to lymphocyte ratio and cardiovascular diseases: a review. Expert Rev Cardiovasc Ther 2013;11(1):55-59. DOI: 10.1586/erc.12.159.

28. Yan Q, Li P, Ye X, et al. Longitudinal peripheral blood transcriptional analysis of COVID-19 patients captures disease progression and reveals potential biomarkers. medRxiv 2020.

29. Jolicoeur $\mathrm{P}$, Lamontagne L. Impairment of bone marrow pre-B and B cells in MHV3 chronically-infected mice. Adv Exp Med Biol 1995;380:193-195.

30. Xu P, Zhou Q, Xu J. Mechanism of thrombocytopenia in COVID-19 patients. Ann Hematol 2020. 1-4.

31. Terpos E, Ntanasis-Stathopoulos I, Elalamy I, et al. Hematological findings and complications of COVID-19. Am J Hematol 2020;95(7): 834-847. DOI: 10.1002/ajh.25829. 